\title{
Special issue on contactless charging for electric vehicles
}

With increasing demands for relieving the pressure on traditional resources and our living environment, the electric vehicle (EV) develops rapidly as a green and sustainable transportation. In the meantime, an effective energy supplement is one of the important technical issues for EVs, which determines its further development and applications. Wireless power transfer (WPT) technologies have become a very hot research topic and attracted increasing attentions, since this emerging technique can enable the power to be transmitted from the supply to the load in a cordless way. Accordingly, WPT technologies can be applied in various industrial and household applications. Especially for EVs, it can be energized flexibly in both static and dynamic ways. Thus, the WPT technique can significantly enhance the driving experiences and performances of EVs by comparing with conventional plug-in chargers. Without awaiting the breakthrough of battery technologies, WPT has shown great potentials to push forward the further development and popularization of EVs.

This special issue includes some specific issues and technologies of WPT for EVs. The aim is to present academic researchers and industrial engineers latest results and findings of WPT technique for EVs, especially on the working principle, electromagnetic field theory, power electronic technique and applications, as well as the future development directions. The special issue is organized in two themes: the first three papers report new researches on electromagnetic analysis, living objects detection and battery wireless charging, and the last paper makes an overview of metamaterial-based WPT technologies for EVs.

The first paper introduces the numerical evaluation of the magnetic field equipped with a WPT system in an EV. A finite element method (FEM) code with a transition boundary condition (TBC) is used to model conductive material. It has been validated to predict the field in an EV made of three different materials: steel, aluminum and fiber composite equipped with a $7.7 \mathrm{~kW}$ WPT system operating at frequencies of 85 or $150 \mathrm{kHz}$, which can be used to assess the safety of EVs using WPT system.

The second paper reports a new approach to prevent living objects from being exposed to the magnetic field by monitoring the air gap under the vehicle underbody utilizing an automotive radar sensor on the vehicle side. Two-dimensional signal processing technologies are applied to detect even the smallest movements of unexpected objects. The method is principally practical and can arouse further researches on its usability.

The third paper reports an experimental analysis in open-loop for lead-acid battery wireless charging using seriesparallel compensation. In charging process an expression of optimum operating frequency is derived to gain the maximum compensated coil efficiency during the load variation. A suitable closed-loop controller can be further realized based on the experimental results and discussion.

The fourth paper reviews recent work on metamaterials and its applications to WPT technologies. By using metamaterials, salient advantages of such WPT systems can be classified into enhancing efficiency, increasing distance, improving misalignment tolerance and compacting size comparing with traditional WPT systems. It also discusses about the development opportunities and technical challenges ahead of metamaterial-based WPT for EVs and illuminate future studies for EV contactless charging systems.

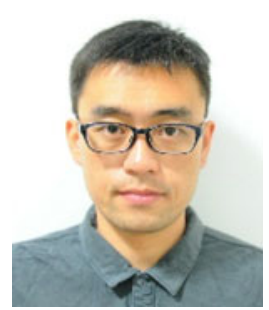

Zhen Zhang (S'11-M'13-SM'15) received the B. Eng. and M. Eng. degrees from Tianjin University, Tianjin, China, in 2004 and 2007, respectively, and the Ph.D. degree from The University of Hong Kong, Hong Kong, in 2014. He worked at IBM Research Laboratory supported by IBM Great Minds Program and served as a Postdoctoral Fellow at The University of Hong Kong in 2014. Currently, he is an Associate Professor in the School of Electrical and Information Engineering at Tianjin University and an Honorary Associate Professor in the Department of Electrical and Electronic Engineering at The University of Hong Kong. He has authored or co-authored more than 30 internationally refereed papers and two book chapters. His research interests include wireless power transfer, electric drives, power electronics, and distributed energies. Dr. Zhang currently serves as an associate editor of the IET Renewable Power Generation, an executive editor of the Cambridge Journal Wireless Power Transfer, and an Editorial Board Member of the IEEE Transactions on Magnetics. 